\title{
Certolizumab in the long-term treatment of rheumatoid arthritis
}

This article was published in the following Dove Press journal:

Open Access Rheumatology: Research and Reviews

25 August 20II

Number of times this article has been viewed

\author{
Abdul Khan \\ David L Scott \\ Department of Rheumatology, King's \\ College London School of Medicine, \\ King's College, London, UK
}

\begin{abstract}
Rheumatoid arthritis is the most common inflammatory arthritis and continues to have major long-term effects on quality of life. Early and intensive treatment has now become the norm in clinical practice with changes of medication dictated by measuring the presence of continued disease activity. Biologics, particular tumor necrosis factor inhibitors, have a crucial role in the management of very severe disease. Certolizumab is a relatively new tumor necrosis factor inhibitor which uses a novel strategy to neutralize TNF-alpha - the conjugation of tumor necrosis factor specific Fab antibody fragments to polyethylene glycol. Two Phase II and three Phase III randomized controlled trials have evaluated the efficacy and toxicity of certolizumab. More than 2000 patients were enrolled, and followed from 12-52 weeks. The number of patients achieving significant improvements with certolizumab, was indicated by the American College of Rheumatology with a 50\% response rate. The risk ratios of achieving this response at 24 weeks was 6.01 (95\% confidence interval [CI]: 3.84-9.40). At 52 weeks the risk ratio was 5.27 (95\% CI: $3.19-8.71)$. The number of patients needed to treat, to obtain this benefit at 24 weeks was 4 (95\% CI: 3-5). Certolizumab also had important clinical benefits in reducing erosive damage to joints, limiting disability, and enhancing other outcomes of importance to patients such as fatigue. The patient-related benefits were present from the early weeks of treatment. The clinical trials showed serious adverse events, including infections, which were more frequent for certolizumab. The most common adverse events comprised upper respiratory tract infections, hypertension, and nasopharyngitis. The balance of evidence suggests that certolizumab is equivalent to other tumor necrosis factor inhibitors, though no head-to-head trials have been undertaken. Having several effective treatments available, benefits patient choice, because the frequency and route of administration of these treatments varies. Furthermore, as intolerance and antibody development against existing biologics is not uncommon, having several agents allows opportunities to switch from one inhibitor to another.
\end{abstract}

Keywords: rheumatoid arthritis, certolizumab, anti-TNF

\section{Introduction}

\section{Features of rheumatoid arthritis}

Rheumatoid arthritis remains the most common inflammatory arthritis. It affects between $0.5 \%$ and $1.0 \%$ of the population. ${ }^{1}$ The incidence of new cases varies from 5 to 50 per 100,000 annually. ${ }^{2}$ Rheumatoid arthritis can begin at any age, including childhood, but the incidence of new cases increases with age. Rheumatoid arthritis has a marked female predominance. Fifty per cent of the risk for development of rheumatoid arthritis is attributable to genetic factors and smoking is the main environmental risk. ${ }^{2}$
Correspondence: Abdul Khan

Department of Rheumatology,

King's College London School of

Medicine, Weston Education Centre,

King's College, London, 10 Cutcombe

Road, London SE5 9RS, UK

$\mathrm{Tel}+4402078485215$

Fax +4402078485202

Email abdulkhanI@nhs.net 
Rheumatoid arthritis results in chronic pain, disability, fatigue, and loss of productivity both in the workplace and at home. Its impact extends beyond chronic pain and inability to function normally. In particular there are significant economic burdens attributable to the disease, which affects society as a whole, as well as individual patients and their families. Work disability in patients with rheumatoid arthritis occurs early and increases over time, and is a major driver of its economic impact. Finally rheumatoid arthritis is associated with cardiovascular disease and a range of other important comorbidities. These shorten life expectancy.

\section{Managing rheumatoid arthritis}

Early and intensive treatment has now become the norm in clinical practice with changes of medication dictated by measuring the presence of continued disease activity. Most patients with rheumatoid arthritis receive treatment with disease-modifying drugs (DMARDs) such as methotrexate and sulfasalazine. These control joint inflammation and are both effective and cost-effective. There is also evidence that combinations of DMARDs are useful in severe, active rheumatoid arthritis.

However, the introduction of biologics has changed the situation. Tumor necrosis factor (TNF) inhibitors are effective at all stages of rheumatoid arthritis. Their benefits extend to early DMARD-naïve rheumatoid arthritis patients. ${ }^{3-5}$ Some expert groups, such as the American College of Rheumatology (ACR), recommend their use as first-line treatment in patients with high disease activity. ${ }^{6}$ However, this remains a controversial issue and other groups, such as the National Institute for Health and Clinical Excellence guidance focuses on DMARD combinations for active early disease, based on an economic analysis of benefits. ${ }^{7,8}$ The combination of adequately dosed methotrexate and a biologic agent, especially a TNF inhibitor, is far more effective in active rheumatoid arthritis than traditional disease modifying drugs used as monotherapy in early and long-standing disease. Biological therapies improve clinical, radiologic, and functional outcomes.

Inevitably, not all patients respond to all medications equally. Some patients respond to one biologic and others respond to another. Some patients may fail a number of medications, either alone or in combination, before responding to an additional treatment. For this reason, the optimal management of rheumatoid arthritis requires access to a range of effective agents. One or two TNF inhibitors are insufficient and there is room for an additional biologic such as certolizumab.
Clinical trials of biologic TNF inhibitors in the late 1990s confirmed the importance of TNF function in the pathogenesis of chronic non-infectious inflammation of joints. Until 2009, more than 2 million patients worldwide had received the first marketed drugs, namely the monoclonal TNF antibodies infliximab and adalimumab and the soluble TNF receptor etanercept..$^{9}$ All three are equally effective in rheumatoid arthritis. These three drugs targeting TNF are now in common clinical use. Infliximab is a chimeric TNF specific monoclonal antibody with mouse hypervariable domains and human antibody backbone. ${ }^{10}$ Adalimumab is a recombinant human TNF specific monoclonal antibody. ${ }^{11}$ Etanercept is a fully human construct comprising the $\mathrm{p} 75 \mathrm{TNF}$ receptor and Fc antibody portion. ${ }^{12}$ The efficacy of these agents in controlling the symptoms of rheumatoid arthritis provides further clinical evidence that in many patients with rheumatoid arthritis, TNF is a central pathogenic mediator.

\section{Structure and function}

Currently, five anti-TNF inhibitors are available to treat rheumatoid arthritis: adalimumab, etanercept, infliximab, golimumab, and certolizumab. Despite having different proposed mechanisms of action, ${ }^{13}$ all five of the current TNF inhibitors have relatively similar effects on clinical, functional, and radiologic outcomes, although there are no head to head trials to confirm their equivalence. The US Food and Drug Administration approved the use of TNF inhibitors to treat rheumatoid arthritis in the following order: etanercept (1998), infliximab (1999), adalimumab (2002), and certolizumab and golimumab (2009).

More biologics are being introduced; as we know, many patients will respond to these TNF inhibitors but some will not, either due to primary failure or secondary TNF failure. Certolizumab (CDP-870) is a new agent that employs a novel strategy to neutralize TNF-alpha, namely the prokaryotic expression of TNF-alpha-specific Fab antibody fragments, conjugated to polyethylene glycol to produce a drug that is potentially less expensive to manufacture than other anti-TNF-alpha agents and which may be administered by subcutaneous injection once a month. ${ }^{14}$

Structurally, there are two important regions, the Fab and the Fc portions. The Fab portion contains complementaritydetermining regions (CDR), unique sequences of amino acids responsible for binding antigen. The Fc portion is not antigen specific but is necessary for other antibody functions including complement fixation and cell lysis. Monoclonal antibodies have a single identical sequence, in contrast to polyclonal antibodies, which have many different sequences 
and hence antigen-binding properties. The first generation of monoclonal antibodies was generated in mice, but the immunogenicity of murine proteins in humans precluded their use therapeutically, due to their tendency to induce major immune responses (anaphylaxis). New techniques have been developed to limit the immunogenicity of monoclonal antibodies, such as "humanization". This involves replacement of murine framework sequences around the CDR with human framework sequences. Certolizumab has been developed using this technique. It consists of only the Fab portion $(50 \mathrm{kD})$ of a monoclonal antibody directed against TNF- $\alpha$, with humanized framework sequences and a $2 \times 20 \mathrm{kD}$ pegol domain. The resulting molecule contains only the smallest effective antigen-binding part of the monoclonal antibody. ${ }^{14}$

Complement or antibody-dependent cell-mediated cytotoxicity, which has been observed in vitro with adalimumab, etanercept, and infliximab, ${ }^{14}$ was not seen in certolizumab as it lacks an Fc region. Certolizumab pegol binds to TNF and prevents its interaction with specific receptors, thus neutralizing it. Certolizumab pegol has been demonstrated to be more potent at neutralizing membrane-bound TNF than etanercept and more potent at neutralizing soluble TNF than adalimumab and infliximab. ${ }^{15}$ It lacks an Fc portion and is therefore unable to fix complement or to lyse cells with surface-bound TNF, in contrast to infliximab and adalimumab. ${ }^{16}$ Certolizumab pegol does not bind to lymphotoxin (TNF $\beta$ ), as it is derived from a monoclonal antibody, in contrast to etanercept. ${ }^{17}$ It is also the only anti-TNF agent that does not kill activated lymphocytes and monocytes by apoptosis or increase levels of degranulation and necrosis of granulocytes in vitro. ${ }^{18}$

\section{Key studies of certolizumab Phase II studies}

The first Phase II study was published in 2002. ${ }^{18}$ It was a double-blinded, randomized, placebo-controlled trial; 36 patients were randomized into two groups. One group received a single intravenous infusion of placebo and the other group received a single ascending dose of 1,5 , or $20 \mathrm{mg} / \mathrm{kg}$ of certoilizumab.

The trial studied patients with severe active disease who had more than three swollen and six tender joints and an erythrocyte sedimentation rate (ESR) of $>28 \mathrm{~mm} /$ hour. The response was measured according to ACR response criteria where an ACR20 indicated a 20\% clinical improvement from baseline after treatment and an ACR50 and ACR70 indicated a 50\% or $70 \%$ improvement, respectively. An ACR20 was designed to show difference between drug and placebo, while ACR50 and ACR70 responses were clinically meaningful to patients. ${ }^{19}$
The study showed a dose dependent response, the $1 \mathrm{mg} / \mathrm{kg}$ dose being no better than placebo, but there was a significant response at the higher doses. The $20 \mathrm{mg} / \mathrm{kg}$ dose showed no clear benefit over the $5 \mathrm{mg} / \mathrm{kg}$ dose in the ACR20 response ( $75 \%$ vs $75 \%$, respectively, at 8 weeks), but did show an increase in the number of patients achieving an ACR50 (50\% and $13 \%$ respectively at 8 weeks). The treatment was well tolerated, with no infusion-related reactions. ${ }^{19}$

A second Phase II study was of subcutaneous certolizumab. It was a randomized, double-blind, placebo-controlled trial. Patients were given 50,100, 200, or $400 \mathrm{mg}$ of certolizumab or placebo subcutaneously every 4 weeks for 12 weeks. The response was measured using the ACR criteria. Patients receiving $400 \mathrm{mg}$ achieved an ACR20 of 60\%, ACR50 of $40 \%$, and ACR70 of $29 \%$ at 12 weeks which showed clear dose response. Patients receiving $400 \mathrm{mg}$ also had an improvement in their health-related quality of life and the drug was well tolerated. ${ }^{19,20}$

\section{Key Phase III trials}

The key Phase III trials comprised RAPID 1, ${ }^{21}$ RAPID 2, ${ }^{22}$ and FAST4WARD. ${ }^{23}$ They explored the use of certolizumab as a monotherapy and also in combination with methotrexate using various dose regimes (Table 1).

RAPID 1 (Rheumatoid Arthritis Prevention of Structural Damage 1) evaluated the efficacy and safety of two doses of certolizumab with methotrexate, or placebo with methotrexate in patients with active rheumatoid arthritis and an inadequate response to methotrexate alone. This 52-week trial involved 982 patients who were randomized to receive subcutaneous certolizumab at an initial dosage of $400 \mathrm{mg}$ given at weeks 0,2 , and 4 , with a subsequent dose of $200 \mathrm{mg}$ or $400 \mathrm{mg}$ given every 2 weeks together with methotrexate, or placebo plus methotrexate. The entry criteria comprised nine or more tender joints, nine or more swollen joints, and an ESR of $30 \mathrm{~mm} /$ hour or more or C-reactive protein (CRP) of $1.5 \mathrm{mg} / \mathrm{dL}$ or more. Disease duration was from 6 months to 15 years (Table 1 ).

At week 24, ACR20 response rates for the certolizumab $200-\mathrm{mg}$ and $400-\mathrm{mg}$ groups were $59 \%$ and $61 \%$ respectively compared with $14 \%$ for the placebo group $(P<0.001$; Table 2). Differences in ACR20 response rates compared with placebo were sustained until 52 weeks $(P<0.001)$.

RAPID 2 was another double-blind, randomized, placebo-controlled study. It enrolled 619 patients with active rheumatoid arthritis who had inadequate responses to methotrexate therapy (Table 1). The patients were randomized 2:2:1 to receive certolizumab $200 \mathrm{mg}$ with methotrexate $(\mathrm{n}=246)$, 
certolizumab $400 \mathrm{mg}$ with methotrexate $(\mathrm{n}=246)$, or placebo with methotrexate $(n=127)$ every 2 weeks for 24 weeks. The primary outcome measure was ACR 20 response at week 24 (Table 2). The ACR20 response at week 24 was achieved in $57 \%, 58 \%$, and $9 \%$ of patients on certolizumab $200 \mathrm{mg}$, $400 \mathrm{mg}$, and placebo respectively ( $P \leq 0.001$; Table 2$)$.

Treatment with certolizumab plus methotrexate was also associated with significant improvement in Disease Activity Score (DAS) 28 (ESR) from baseline vs placebo. At week 24 the mean changes were $200 \mathrm{mg}-2.27$ (SD 1.38), $400 \mathrm{mg},-2.46$ (SD 1.31) and placebo -0.50 (SD 1.05). DAS28 remission (with DAS28 scores under 2.6) was seen in $9 \%$ of patients treated with certolizumab $200 \mathrm{mg}$ or $400 \mathrm{mg}$ respectively at week 24 , compared with $1 \%$ of patients in the placebo group $(P<0.05$; Figure 1$)$.

The final trial, FAST4WARD, was a 24-week, randomized, double-blind, placebo-controlled study evaluating certolizumab as monotherapy in 220 patients who had previously failed one or more DMARDs. Patients were aged 18 to 75 years, and had adult onset rheumatoid arthritis by the 1987 ACR criteria ${ }^{24}$ of $>6$ months duration. Disease duration was from 6 months to 15 years. Disease activity entry criteria were the same as RAPID 1 and RAPID 2 (Table 1).

Patients were randomized to receive subcutaneous certolizumab $400 \mathrm{mg}(\mathrm{n}=111)$ or placebo $(\mathrm{n}=109)$ every 4 weeks. The primary end-point was ACR20 response rates at week 24 . This was achieved by $46 \%$ of the certolizumab group and $9 \%$ of the placebo group $(P<0.001)$. ACR50 and ACR70 at week 24 , using non-responder imputation, were significantly higher for certolizumab than placebo ( $23 \%$ vs $4 \%, P<0.001$ and $6 \%$ vs none, $P<0.05$ respectively; Table 2 ).

\section{Effect on function}

At week 24 significantly more patients in the certolizumab treatment groups reported improvements in patient reported outcomes (PROs) including fatigue measured by the Fatigue Assessment Scale (FAS), arthritis pain measured on a visual analog scale (VAS) and physical function measured using the Health Assessment Questionnaire (HAQ) ${ }^{25}$ The beneficial effects of certolizumab were similar between the $200 \mathrm{mg}$ and $400 \mathrm{mg}$ dose levels with no significant difference between treatment groups in any PROs ${ }^{25}$ (Table 3).

In addition at week $2463 \%$ of certolizumab $200 \mathrm{mg}$ treated patients reported clinically meaningful improvements in one or more PROs compared with 13\% in placebo groups. Approximately $23 \%$ of all certolizumab-treated patients reported clinically meaningful improvements in all three PROs compared with $3 \%$ in placebo groups. ${ }^{23}$ 
Table 2 American College of Rheumatology responders in key trials

\begin{tabular}{|c|c|c|c|c|c|c|c|}
\hline Trial & Year & Endpoint & Group & Patients & ACR20 & ACR50 & ACR70 \\
\hline \multirow{3}{*}{ RAPID I ${ }^{20}$} & 2008 & 24 weeks & Certolizumab 400 mg & 390 & $60.8 \%$ & $39.9 \%$ & $20.6 \%$ \\
\hline & & & Certolizumab $200 \mathrm{mg}$ & 393 & $58.8 \%$ & $37.1 \%$ & $21.4 \%$ \\
\hline & & & Placebo & 199 & $13.6 \%$ & $7.6 \%$ & $3.0 \%$ \\
\hline \multirow[t]{3}{*}{ RAPID $2^{21}$} & 2008 & 24 weeks & Certolizumab $400 \mathrm{mg}$ & 246 & $57.3 \%$ & $32.5 \%$ & $15.9 \%$ \\
\hline & & & Certolizumab 200 mg & 246 & $57.3 \%$ & $32.5 \%$ & $15.9 \%$ \\
\hline & & & Placebo & 127 & $8.7 \%$ & $3.1 \%$ & $0.8 \%$ \\
\hline \multirow[t]{2}{*}{ FAST4WARD ${ }^{22}$} & 2009 & 24 weeks & Certolizumab $400 \mathrm{mg}$ & 111 & $45.5 \%$ & $22.7 \%$ & $5.5 \%$ \\
\hline & & & Placebo & 109 & $9.3 \%$ & $3.7 \%$ & $0 \%$ \\
\hline
\end{tabular}

Abbreviation: ACR, American College of Rheumatology.

Significant reductions in fatigue were reported by patients receiving certolizumab monotherapy compared with placebo at Week $1(P<0.01)$ and were sustained to week 24 $(P<0.001$; Table 3$)$. Meaningful reductions in fatigue were reported by $46 \%$ of certolizumab patients compared with $17 \%$ of placebo patients $(P<0.001)$.

Patients treated with certolizumab $400 \mathrm{mg}$ monotherapy reported statistically significant improvements in physical function assessed by HAQ compared with patients receiving placebo from week 1 ( -0.23 vs 0.04 , respectively) through week 24 ( -0.36 vs $0.13 ; P<0.001$ for both time points) (Table 3). By study end point at week $24,49 \%$ of patients receiving certolizumab reported clinically meaningful improvements in physical function compared with $12 \%$ of those receiving placebo $(P<0.001)$.

\section{Efficacy of certolizumab in systematic reviews}

A Cochrane review published in 2009 assessed the effectiveness and safety of certolizumab in patients with rheumatoid arthritis who had not responded well to conventional DMARDs. ${ }^{26} \mathrm{It}$ evaluated randomized controlled trials that compared certolizumab with placebo or methotrexate in adult patients with active rheumatoid arthritis despite current or prior treatment with conventional DMARDs, such as methotrexate.

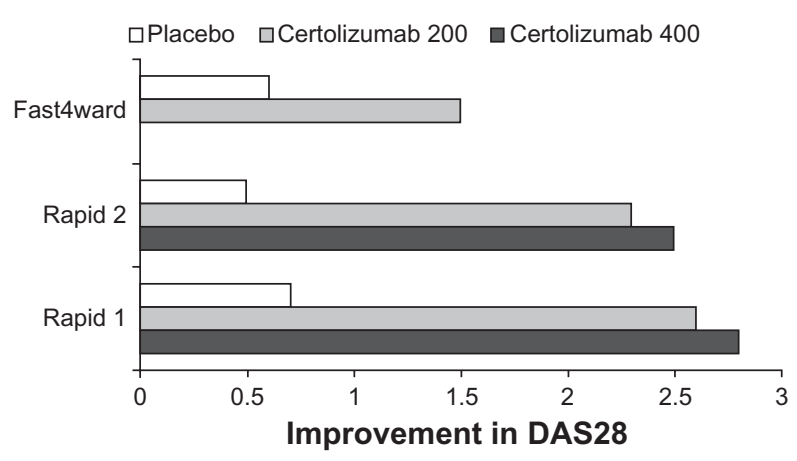

Figure I Mean changes in DAS28 in key trials. Abbreviation: DAS, Disease Activity Score.
Five trials were included. The systematic review analyzed 2394 people for effectiveness. The duration of follow-up was from 12 to 52 weeks, and the doses of certolizumab pegol ranged from 50 to $400 \mathrm{mg}$ subcutaneously. In three trials the control was placebo plus methotrexate ${ }^{21-23}$ and in two trials it was just placebo. ${ }^{19,20}$

Significant improvements were observed at 24 weeks with the approved dose of $200 \mathrm{mg}$ certolizumab. ACR50 risk ratios (RR) were 6.01 (95\% CI: 3.84-9.40) with an absolute benefit of $29 \%$ (95\% CI: $25 \%-34 \%$ ). The number needed to treat to obtain benefit (NNTB) was 4 (95\% CI: 3-5) and the HAQ mean difference was -0.39 ( $95 \%$ CI: $-0.45-0.32$ ).

At 52 weeks the results were quite similar: ACR50 RRs were 5.27 (95\% CI: 3.19-8.71), HAQ mean difference -0.42 (95\% CI: $-0.52--0.32$ ).

A comparative meta-analysis against other anti-cytokine agents has been undertaken by Launois et al. ${ }^{27}$ In their metaanalysis, using the random-effects model which takes heterogeneity into account, certolizumab showed the highest odds ratio (OR) for ACR20 responses (OR: 11.82, 95\% CI 5.98-21.71). Certolizumab also had the highest OR for ACR50 and ACR70 responses. In the main, the differences in ORs between different anti-cytokine treatments did not achieve statistical significance, but are an interesting finding, which might represent either a treatment benefit, trial design, or both.

\section{Effects on erosive damage}

Radiographic damage is believed to start early in the disease course of rheumatoid arthritis ${ }^{28}$ and therefore early intervention is vital. ${ }^{29}$ The widespread use of biologics has necessitated improved outcome measures. TNF inhibitors have changed the therapeutic goals in rheumatoid arthritis not only by relieving signs and symptoms but also by inducing remission. The value of structural damage and physical function as outcome measures is increasingly recognized, since damage is related to function, which affects quality of life.$^{30}$ Radiographic damage in patients with rheumatoid 
Table 3 Changes in fatigue, pain, and disability in key trials

\begin{tabular}{lllll}
\hline Trial & Outcome & Placebo & Certolizumab 200 mg & Certolizumab 400 mg \\
\hline RAPID I (week 52) & Fatigue (FAS) & $-0.8(0.2)$ & $-2.6(0.1)^{*}$ & $-2.5(0.1)^{*}$ \\
& Arthritis pain (VAS) & $-8.8(I .6)$ & $-31.0(1.2)^{*}$ & $-33.5(I .2)^{*}$ \\
& Physical function (HAQ-DI) & $-0.18(0.04)$ & $-0.60(0.03)^{*}$ & $-0.63(0.03)^{*}$ \\
RAPID 2 (week 24) ${ }^{21}$ & Fatigue (FAS) & $-0.5(0.2)$ & $-2.0(0.1)^{*}$ & $-2.2(0.1)^{*}$ \\
& Arthritis pain (VAS) & $-4.7(1.9)$ & $-23.7(I .4)^{*}$ & $-26.1(1.4)^{*}$ \\
FAST4WARD (week 24) & Physical function (HAQ-DI) & $-0.14(0.04)$ & $-0.50(0.03)^{*}$ & $-0.50(0.03)^{*}$ \\
& Fatigue (FAS) & -0.3 & - & $-1.7^{*}$ \\
& Arthritis pain (VAS) & 1.7 & - & $-20.6^{*}$ \\
& Physical function (HAQ-DI) & 0.13 & - & $-0.36^{*}$ \\
\hline
\end{tabular}

Notes: Mean changes (standard deviations) are shown; $* P<0.001$ compared with placebo.

Abbreviations: FAS, Fatigue Assessment Scale; VAS, visual analog scale; HAQ-DI, Health Assessment Questionnaire Disability Index.

arthritis has become an important outcome measure in clinical trials. An association between disease activity and radiographic damage has been demonstrated in several studies. ${ }^{31-33}$ Radiographs of hands and feet are easy and inexpensive to perform, and standard methodologies are available to measure progression. They are assessed using the Sharp/van der Heijde method ${ }^{34}$ or the Larsen method, ${ }^{35}$ which quantifies erosions and joint space narrowing. RAPID 1 evaluated the efficacy and safety of certolizumab in combination with methotrexate. A decrease in radiographic progression was evident at 52 weeks for both dosage regimens, despite the fact that $60 \%$ of the patients in the placebo group withdrew at week 16 and entered the open-label certolizumab treatment. ${ }^{22}$

RAPID 2 followed the same dosage regimen as RAPID 1, but the primary end-point was ACR20 at week 24 in a population of 619 patients with rheumatoid arthritis. Radiographic findings in RAPID 2 showed that in patients treated with certolizumab $200 \mathrm{mg}$ the mean changes from baseline in the van der Heijde modified Total Sharpe Score at week 24 in the $200 \mathrm{mg}$ certolizumab, $400 \mathrm{mg}$ certolizumab, and placebo plus methotrexate groups were $0.2,-0.4$, and 1.2 respectively. Changes on certolizumab treatment were both significantly less than those with placebo and methotrexate $(P<0.01)$.

Certolizumab plus methotrexate therapy inhibited the progression of structural damage to a greater extent than placebo plus methotrexate therapy. At week 52, mean radiographic progression from baseline was reduced in patients treated with certolizumab $200 \mathrm{mg}$ (0.4 Sharp units) or $400 \mathrm{mg}$ ( 0.2 Sharp units) compared with that in placebotreated patients (2.8 Sharp units) $(P<0.001$; Figure 2$)$. The trial showed that the drug had slowed mean radiographic progression from baseline by week 52 and improved physical function as early as week 1 .

\section{Economic considerations}

Kavanaugh et al undertook an evaluation of the economic outcomes in the RAPID 1 and RAPID 2 trials. ${ }^{36}$ At baseline $40 \%$ to $42 \%$ of patients were employed outside the home. Certolizumab significantly reduced work absenteeism and presenteeism among patients working outside the home compared with placebo. It also significantly reduced the number of household days lost, household days with productivity reduced by $50 \%$ or more, and lost participation in family, social, and leisure activities. Improvements were seen by 4 weeks and continued for up to 12 months.

Detailed cost-effective analyses have been undertaken in the UK in research commissioned by the National Institute For Health and Clinical Excellence. ${ }^{37}$ As a number of different analytical approaches have been used there is a range of different conclusions, depending on the assumptions made in the various models. The most useful analysis is probably the probabilistic sensitivity analysis. This showed that the probability of certolizumab pegol with methotrexate being cost effective at a willingness-to-pay threshold of $£ 20,000$ per

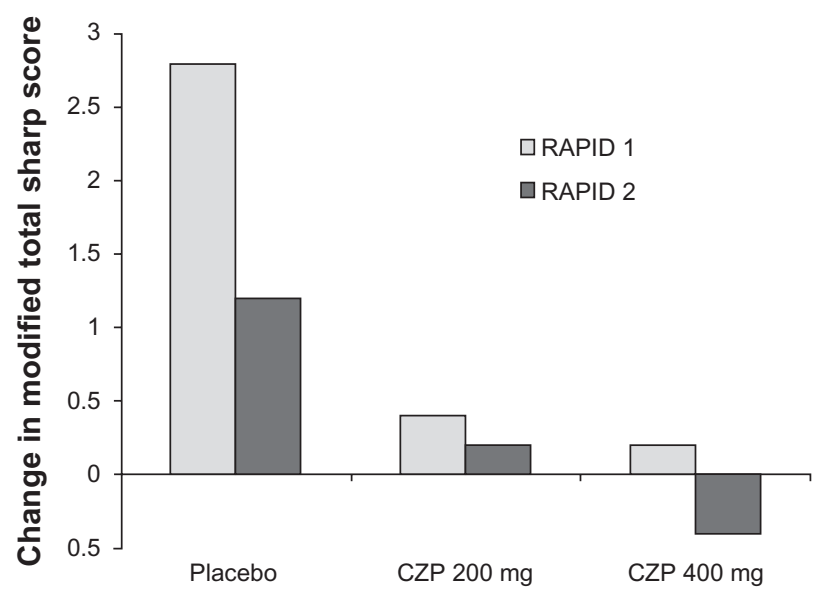

Figure 2 Mean changes in radiographic scores in key trials. Abbreviation: CZP, certolizumab pegol. 
quality adjusted life-year gained was $49 \%$. The probability of certolizumab monotherapy being cost effective at $£ 20,000$ per quality adjusted life-year gained was $46 \% .^{37}$

\section{Adverse events}

The adverse events reported in the key clinical trials ${ }^{21-23}$ are summarized in Table 4 . The majority of adverse events were mild or moderate. Over half the patients in both treatment and placebo groups reported one or more adverse event. However, serious adverse events were seen in about $3 \%$ of placebo-treated patients and about $7 \%$ of patients receiving different doses of certolizumab.

Adverse events reported in more than $5 \%$ of patients taking certolizumab included headache, nasopharyngitis, upper respiratory tract infections, diarrhea, and sinusitis. ${ }^{20-22}$ A range of serious adverse events were reported with certolizumab including bacterial arthritis, mastitis, benign parathyroid tumor, postural dizziness, ischemic stroke, and menorrhagia. Overall, within the limited duration of exposure in the trials, the adverse event profile for certolizumab was comparable with other TNF inhibitors. ${ }^{21-23}$

A systematic review of trials of certolizumab has evaluated safety in 2094 people in five trials. ${ }^{25}$ Serious adverse events were more frequent for certolizumab pegol $200 \mathrm{mg}$ (Peto OR: 2.02, 95\% CI: 1.24-3.30). The most common adverse events with certolizumab $200 \mathrm{mg}$ were upper respiratory tract infections (Peto OR: 2.21, 95\% CI: 1.15-4.25); hypertension (Peto OR: 2.81, 95\% CI: 1.38-5.75), and nasopharyngitis (Peto OR: 2.71, 95\% CI: 1.30-5.66).

Finally, a network meta-analysis by Singh et $\mathrm{al}^{38}$ compared adverse effects with a number of TNF inhibitors (etanercept, adalimumab, infliximab, golimumab, and certolizumab), interleukin (IL)-1 antagonist (anakinra), IL-6 antagonist (tocilizumab), anti-CD28 (abatacept), and anti-B cell (rituximab) therapy in patients with any disease condition except human immunodeficiency disease. This network meta-analysis used mixed-effects logistic regression, and an arm-based, random-effects model within an empirical Bayes framework. This analysis included 163 trials with 50,010 patients and 46 extension studies with a further 11,954 patients. After adjusting for dose, biologics as a group were associated with a statistically significant higher rate of total adverse events (OR: 1.19, 95\% CI: 1.09-1.30). The rates of serious adverse events, serious infections, lymphoma, and congestive heart failure were not statistically different between biologics and control treatment. However, certolizumab was associated with significantly higher risk of serious infections compared with control treatment (OR: 3.51, 95\% CI: 1.59-7.79).

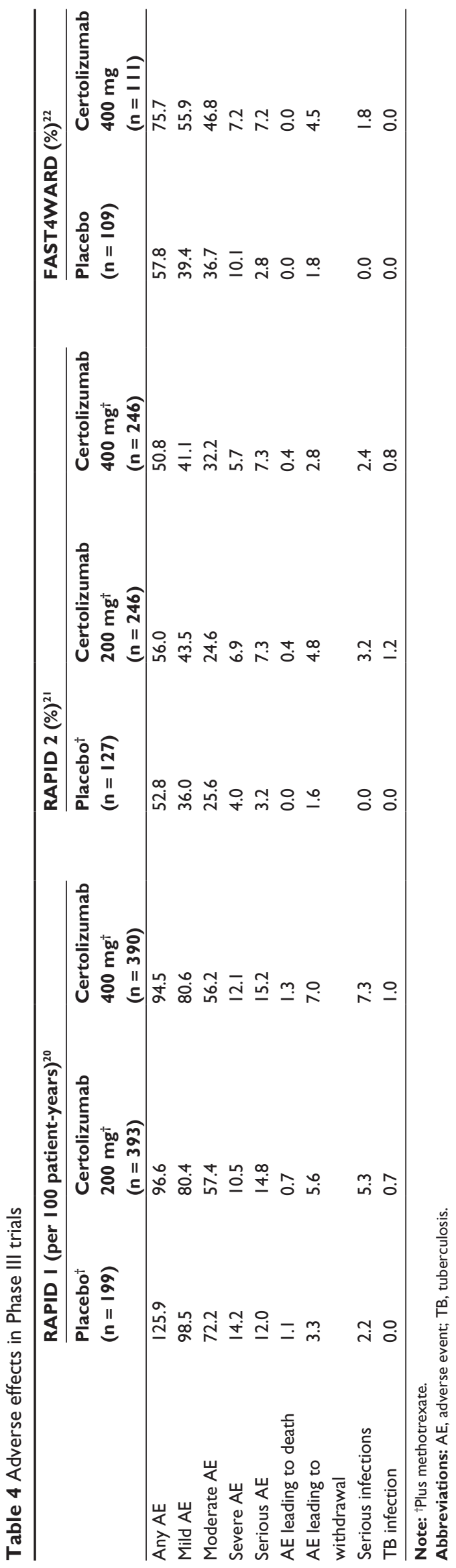




\section{Conclusions}

There is strong evidence from large clinical trials that certolizumab combined with methotrexate or used as a monotherapy improves the signs and symptoms of rheumatoid arthritis within 1-2 weeks of treatment starting. It also inhibits the progression of structural joint damage within 4 months of treatment starting. Finally it improves physical function and reduces pain and fatigue within the first few weeks of treatment. The trials show these benefits are sustained for 12 months and it is likely that they continue beyond 1 year. Certolizumab increases the number of adverse events with statistically significant increases in the number of serious adverse events, infections, and hypertension. There are limited data about its long-term safety but currently there are no causes for concern.

The systematic reviews show some evidence that certolizumab may have both greater efficacy in terms of ACR responders and greater toxicity in terms of serious infections. There are several explanations for these two findings but the most likely one is differences in trial design and patients studied. The differences are small and the balance of evidence suggests that the five available TNF inhibitors are similar with no clear reason to choose one over another. ${ }^{39}$ However, there are several reasons why it is advantageous to have several TNF inhibitors available. Firstly it increases patient choice, as the frequency and route of administration varies. Secondly, as intolerance and antibody development against existing biologics is not uncommon, it offers opportunity to switch from one TNF inhibitor to another. There is evidence that this is useful when patients have adverse effects or treatment failure. ${ }^{40-42}$

Certolizumab has structural characteristics which may theoretically confer advantages. Since it consists only of the Fab component of an antibody and lacks the Fc portion, it cannot fix complement or lyse cells with surface-bound TNF- $\alpha$, unlike infliximab and adalimumab. ${ }^{12}$ It is also the only TNF inhibitor that does not induce apoptosis of activated lymphocytes and monocytes or degranulation and loss of neutrophil viability in vitro. ${ }^{43,44}$ The Fab component is bound to a PEG moiety, which increases its half-life and may reduce the likelihood of anti-drug antibody formation compared with conventional chimeric monoclonal antibodies. ${ }^{43,44}$ Reduced immunogenicity, due to humanization and PEGylation, may result in a decreased likelihood of severe allergic reactions, and also reduced development of neutralizing antibodies. ${ }^{44}$

It is too early to say how certolizumab will eventually be used to treat rheumatoid arthritis. We believe there is a place for another TNF inhibitor, and have presented the arguments in its favor. However, there is a diversity of opinion with some commentators taking more negative views. ${ }^{45}$ While there is no evidence that certolizumab is markedly more effective than other TNF inhibitors, the presence of several effective agents is likely to focus attention on their relative costs; and continuing downward pressures on their costs should have substantial positive benefits on their relative cost-effectiveness.

\section{Disclosures}

Abdul Khan has received no external grants or personal funding from any industrial sponsor. David L Scott receives grant funding support from Arthritis Research UK and The National Institute for Health Research. In the past 3 years Professor Scott has received no personal funding from any industrial sponsor.

\section{References}

1. Kvein TK. Epidemiology and burden and illness of rheumatoid arthritis. Pharmacoeconomics. 2004;22(2 Suppl 1):1-12.

2. Scott DL, Wolfe F, Huizinga TW. Rheumatoid arthritis. Lancet. 2010;376:1094-1108.

3. Bathon JM, Martin RW, Fleischmann RM, et al. A comparison of etanercept and methotrexatein patients with early rheumatoid arthritis. N Engl J Med. 2000;343:1586-1593.

4. St Clair EW, van der Heijde DM, Smolen JS, et al. Combination of infliximab and methotrexate therapy for early rheumatoid arthritis. Arthritis Rheum. 2004;50:3432-3443.

5. Breedveld FC, Weisman MH, Kavanaugh AF, et al. A multicenter, randomised, double-blind clinical trial of combination therapy with adalimumabplus methotrexate versus methotrexate alone or adalimumab alone in patients with early, aggressive rheumatoid arthritis who had not had previous methotrexate treatment. Arthritis Rheum. 2006;54:26-37.

6. Saag KG, Teng GG, Patkar NM, et al. American College of Rheumatology 2008 recommendations for the use of nonbiologic and biologic disease-modifying antirheumatic drugs in rheumatoid arthritis. Arthritis Rheum. 2008;59:762-784.

7. Deighton C, O'Mahony R, Tosh J, Turner C, Rudolf M; Guideline Development Group. Management of rheumatoid arthritis: summary of NICE guidance. BMJ. 2009;338:702.

8. Tosh JC, Wailoo AJ, Scott DL, Deighton CM. Cost-effectiveness of combination nonbiologic disease-modifying antirheumatic drug strategies in patients with early rheumatoid arthritis. $J$ Rheumatol. 2011;38:1593-1600.

9. Sfikakis PP. The first decade of biologic TNF antagonists in clinical practice: lessons learned, unresolved issues and future directions. Curr Dir Autoimmun. 2010;11:180-210.

10. Scallon BJ, Moore MA, Trinh H, et al. Chimeric anti-TNF-alpha monoclonal antibody cA2 binds recombinant transmembrane TNF-alpha and activates immune effector functions. Cytokine. 1995;7:251-259.

11. Mease P. Adalimumab in the treatment of arthritis. Ther Clin Risk Manag. 2007;3:133-148.

12. Danila MI, Hughes LB, Bridges SL. Pharmacogenetics of etanercept in rheumatoid arthritis. Pharmacogenomics. 2008;9:1011-1015.

13. Wong M, Ziring D, Korin D, et al. TNF $\alpha$ blockade in human diseases: mechanisms and future directions. Clin Immunol. 2008;126:121-136.

14. Nesbitt A, Fossati G, Bergin M, et al. Mechanism of action of certolizumab pegol (CDP870): in vitro comparison with other anti-tumor necrosis factor alpha agents. Inflamm Bowel Dis. 2007;13:1323-1332. 
15. Gramlick A, Fossati G, Henry A, et al. Assessment of the affinity for soluble TNF and the neutralising potency against soluble and membrane TNF of the anti-TNF agents certolizumab pegol, adalimumab, etanercept and infliximab. Ann Rheum Dis. 2006;65:456.

16. Fossati $\mathrm{G}, \mathrm{Nesbitt} A$. In vitro complement-dependent cytotoxicity and antibody-dependent cellular cytotoxicity by the anti-TNF agents certolizumab pegol, adalimumab, etanercept and infliximab. Ann Rheum Dis. 2006;65:455.

17. Mpofu S, Fatima F, Moots RJ. Anti-TNF-alpha therapies: they are all the same (aren't they?) Rheumatology. 2005;44:271-273.

18. Fossati G, Nesbitt A. Effect of the anti-TNF agents certolizumab pegol, adalimumab, etanercept and infliximab on levels of apoptosis in activated peripheral blood lymphocytes and monocytes and on necrosis and degranulation of peripheral blood granulocytes. Ann Rheum Dis. 2006;65:622.

19. Choy EH, Hazleman B, Smith M, et al. Efficacy of a novel PEGylated humanized anti-TNF fragment (CDP870) in patients with rheumatoid arthritis: a phase II double-blinded, randomized, dose-escalating trial Rheumatology. 2002;41:1133-1137.

20. Kaushik V, Moots R. CDP-870 (certolizumab) in rheumatoid arthritis. Expert Opin Biol Ther. 2005;5:601-606.

21. Keystone E, Heijde D, Mason D Jr, et al. Certolizumab pegol plus methotrexate is significantly more effective than placebo plus methotrexate in active rheumatoid arthritis: findings of a fifty-two-week phase III, multicenter, randomized, double-blind, placebo-controlled, parallel-group study. Arthritis Rheum. 2008;58:3319-3329.

22. Smolen J, Landewe RB, Mease P, et al. Efficacy and safety of certolizumab pegol plus methotrexate in active rheumatoid arthritis: the RAPID 2 study. A randomised controlled trial. Ann Rheum Dis. 2009;68:797-804.

23. Fleischmann R, Vencovsky J, van Vollenhoven R, et al. Efficacy and safety of certolizumab pegol monotherapy every 4 weeks in patients with rheumatoid arthritis failing previous disease-modifying antirheumatic therapy: the FAST4WARD study. Ann Rheum Dis 2009;68:805-811.

24. Arnett F, Edworthy S, Bloch D, et al. The American Rheumatism Association 1987 revised criteria for the classification of rheumatoid arthritis. Arthritis Rheum. 1988;31:315-324.

25. Strand V, Smolen J, Vollenhoven R, et al. Certolizumab pegol plus methotrexate provides broad relief from the burden of rheumatoid arthritis: analysis of patient-reported outcomes from the RAPID 2 trial. Ann Rheum Dis. 2011;70:996-1002.

26. Ruiz Garcia V, Jobanputra P, Burls A, et al. Certolizumab pegol (CDP870) for rheumatoid arthritis in adults. Cochrane Database Syst Rev. 2011;2:CD007649.

27. Launois R, Avouac B, Berenbaum F, et al. Comparison of certolizumab pegol with other anticytokine agents for treatment of rheumatoid arthritis: a multiple-treatment Bayesian metaanalysis. J Rheumatol. 2011;38:835-845.

28. Fuchs HA, Kaye JJ, Callahan LF, Nance EP, Pincus T. Evidence of significant radiographic damage in rheumatoid arthritis within the first 2 years of disease. J Rheumatol. 1989;16:585-591.
29. Lindqvist E, Jonsson K, Saxne T, Eberhardt K. Course of radiographic damage over 10 years in a cohort with early rheumatoid arthritis. Ann Rheum Dis. 2003;62:611-616.

30. Kavanaugh A, Han C, Bala M. Functional status and radiographic joint damage are associated with health economic outcomes in patients with rheumatoid arthritis. J Rheumatol. 2004;31:849-855.

31. Jansen LM, van der Horst-Bruinsma IE, van Schaardenburg D, et al. Predictors of radiographic joint damage in patients with early rheumatoid arthritis. Ann Rheum Dis. 2001;60:924-927.

32. Landewe R, van der Heijde D. Radiographic progression in rheumatoid arthritis. Clin Exp Rheumatol. 2005;23:S63-S68.

33. Van der Heijde DM, van Riel PL, Nuver-Zwart IH, et al. Effects of hydroxychloroquine and sulphasalazine on progression of joint damage in rheumatoid arthritis. Lancet. 1989;1:1036-1038.

34. Van der Heijde D. How to read radiographs according to the Sharp/van der Heijde method. J Rheumatol. 1999;26:743-745.

35. Larsen A, Dale K, Eek M. Radiographic evaluation of rheumatoid arthritis and related conditions by standard reference films. Acta Radiol Diagn (Stockh). 1977;18:481-491.

36. Kavanaugh A, Smolen JS, Emery P, et al. Effect of certolizumab pegol with methotrexate on home and work place productivity and social activities in patients with active rheumatoid arthritis. Arthritis Rheum. 2009;61:1592-1600.

37. Connock M, Tubeuf S, Malottki K, et al. Certolizumab pegol for the treatment of rheumatoid arthritis. Health Technol Assess. 2010; 14(Suppl 2):1-10.

38. Singh JA, Wells GA, Christensen R, et al. Adverse effects of biologics: a network meta-analysis and Cochrane overview. Cochrane Database Syst Rev. 2011;2:CD008794.

39. Scott DL, Cope A. New tumour necrosis factor inhibitors for rheumatoid arthritis: are there benefits from extending choice? Ann Rheum Dis. 2009;68:767-769.

40. Hyrich KL, Lunt M, Watson KD, et al. Outcomes after switching from one anti-tumor necrosis factor alpha agent to a second anti-tumor necrosis factor alpha agent in patients with rheumatoid arthritis: results from a large UK national cohort study. Arthritis Rheum. 2007;56:13-20.

41. Hyrich KL, Lunt M, Dixon WG, et al. Effects of switching between anti-TNF therapies on HAQ response in patients who do not respond to their first anti-TNF drug. Rheumatology. 2008;47:1000-1005.

42. Lloyd S, Bujkiewicz S, Wailoo AJ, Sutton AJ, Scott D. The effectiveness of anti-TNF-alpha therapies when used sequentially in rheumatoid arthritis patients: a systematic review and meta-analysis. Rheumatology. 2010;49:2313-2321

43. Nesbitt A, Fossati G, Brown D. Effect of structure of conventional anti-TNFs and certolizumab pegol on mode of action in rheumatoid arthritis. Ann Rheum Dis. 2007;66:296.

44. Barnes T, Moots R. Targeting nanomedicines in the treatment of rheumatoid arthritis: focus on certolizumab pegol. Int J Nanomedicine. 2007;2:3-7.

45. Certolizumab pegol and rheumatoid arthritis. Just another TNF alpha antagonist, no therapeutic advantage. Prescrire Int. 2010;19:279.

\section{Publish your work in this journal}

Open Access Rheumatology Research and Reviews is an international, peer-reviewed, open access journal, publishing all aspects of clinical and experimental rheumatology in the clinic and laboratory including the following topics: Pathology, pathophysiology of rheumatological diseases; Investigation, treatment and management of rheumatological

\section{Dovepress}

diseases; Clinical trials and novel pharmacological approaches for the treatment of rheumatological disorders. The manuscript management system is completely online and includes a very quick and fair peerreview system, which is all easy to use. Visit http://www.dovepress.com/ testimonials.php to read real quotes from published authors. 